Pacific Journal of Mathematics

ON THE NUMBER OF REAL ROOTS OF POLYNOMIALS 


\title{
ON THE NUMBER OF REAL ROOTS OF POLYNOMIALS
}

\section{Thomas Craven and George Csordas}

\begin{abstract}
A general theorem concerning the structure of a certain real algebraic curve is proved. Consequences of this theorem include major extensions of classical theorems of Pólya and Schur on the reality of roots of polynomials.
\end{abstract}

1. Introduction and definitions. Throughout this paper, all polynomials are assumed to have real coefficients. In 1916, George Pólya wrote a paper [6] in which he considered two polynomials, $f(x)=\sum_{i=0}^{m} a_{i} x^{i}, a_{m} \neq 0$, and $h(x)=\sum_{i=0}^{n} b_{i} x^{i}, b_{n} \neq 0$, where $n \geqq m$, both polynomials have only real roots and the roots of $h$ are all negative. Under these hypotheses, he proved the following theorem.

THEOREM 1.1 (Pólya [6]). The real algebraic curve $F(x, y) \equiv$ $b_{0} f(y)+b_{1} x f^{\prime}(y)+\cdots+b_{m} x^{m} f^{(m)}(y)=0$ has $m$ intersection points with each line $s x-t y+u=0$, where $s \geqq 0, t \geqq 0, s+t>0$ and $u$ is real.

Pólya then noted that this theorem gives a unified proof of three important special cases regarding composite polynomials:

(1.2)(a). Setting $x=1$ gives a special case of the HermitePoulain theorem [5, Satz 3.1].

(1.2)(b). Setting $y=0$ gives a theorem of Schur [5, Satz 7.4].

(1.2)(c). Setting $x=y$ gives a theorem of Schur and Pólya [7, p. 107].

Our main theorem, proved in $\S 2$ establishes some of the properties of $F(x, y)=0$ when we drop all restrictions on $f$ and require only that all the roots of $h$ be real (of arbitrary sign). In the last section, we apply this theorem to obtain an extension of Theorem 1.1 which states that, for $h$ restricted as in Pólya's theorem, there are at least as many intersection points as the number of real roots of $f$. As corollaries, we then obtain the full strength of the Hermite-Poulain theorem and extensions of the theorems of Pólya and Schur to arbitrary polynomials $f$. Pólya states that his theorem is one of the most general known theorems on the reality of roots of polynomials. We believe this statement is still true 
today, more than sixty years later, especially for our much more general version of it. The generalizations that this provides of (1.2)(b) and (c) are used in [2] to solve an open problem of Karlin on characterizing zero-diminishing transformations which he says "seems to be very difficult." They also are used in [1] for many further applications to polynomials and entire functions. For the known results on theorems of this type, the reader is referred to [4] and [5].

Because the subject of algebraic geometry deals almost exclusively with algebraically closed fields, we have found it necessary to develop some new terminology. As much as possible, we follow Walker's book "Algebraic Curves" [8]. In particular, we use the word component to refer to an irreducible factor of the curve $F(x, y)$. Since we deal only with the real points of a (not necessarily irreducible) algebraic variety, we need a term to refer to the individual parts of the curve $F(x, y)=0$, even though these parts may not be components, or even connected components since two parts may intersect. For this we shall use the word branch. Classically, branches are only defined in a neighborhood of a point on the curve [3, p. 39] where they have the usual analytic meaning. We shall use the word branch in the following global sense: the branch of the curve $F$ containing a given point on the curve is obtained by travelling along the curve in both directions until reaching a singularity or returning to the starting point; at a singularity travel out along the other arc of the same local branch on which you arrived. Thus the branches are the "pieces" into which a circuit in the projective plane $[3$, p. 50] is broken by removing the line at infinity. We shall see in the next section that the branches in which we are most interested will always go to infinity in both directions. In particular, if $f$ has simple roots, the curve $F(x, y)$ in Theorem 1.1 is nonsingular and the branches are just the connected components. As is usual in algebraic geometry, all roots, branches and components will be counted including multiplicities, with the exception of Lemma 2.1.

2. The main theorem. We begin with a lemma which describes the variety $F(x, y)=0$ when the polynomial $h(x)$ has degree one. It will be used in an inductive step in the proof of the main theorem.

LEMmA 2.1. Let $f(y)=\sum_{i=0}^{n} a_{i} y^{i}, a_{n} \neq 0$, be any polynomial with real coefficients and let $\alpha$ be any nonzero real number. Set $F(x, y)=\alpha f(y)+x f^{\prime}(y)$. Assume that $f$ has $r$ real roots $\alpha_{1}, \cdots, \alpha_{r}$ and that $f^{\prime}$ has $s$ real roots $\beta_{1}, \cdots, \beta_{s}$, listed with multiplicity if 
and only if they are also roots of $f$. Then the real variety $F(x, y)=0$ satisfies the following;

(1) The variety consists of $s+1$ one-dimensional branches (not necessarily distinct), exactly $r$ of which cross the $y$-axis.

(2) The branches are disjoint unless $f$ has a multiple real root. At a root $\alpha_{i}$ of multiplicity $k$, we have $k-1$ components coinciding with the line $y=\alpha_{i}$ and one other branch through $\left(0, \alpha_{i}\right)$. Other than coincidental lines $y=\alpha_{i}$, branches can only intersect on the $y$-axis.

(3) Each branch which intersects the $y$-axis will intersect it only once and will also intersect each line $x=c$, where $c$ is a constant.

(4) All branches are asymptotic to straight lines from the set $y=\beta_{i}, i=1,2, \cdots, s$ and $y=-n \alpha^{-1} x-a_{n-1} a_{n}^{-1}$.

Proof. Since $F(x, y)=\alpha f(y)+x f^{\prime}(y)=0$, we can write

$$
x=-\frac{\alpha f(y)}{f^{\prime}(y)} \text { whenever } f^{\prime}(y) \neq 0 .
$$

If $f$ has a root $\alpha_{i}$ of multiplicity $k$, then $F(x, y)$ has a factor of $\left(y-\alpha_{i}\right)^{k-1}$. Canceling this from the numerator and denominator in (2.2) shows that there is still a branch passing through $\left(0, \alpha_{i}\right)$. Other than this situation, it is clear from (2.2) that the branches are all disjoint so that claim (2) of the lemma holds. From (2.2) we also see that for large values of $y$, the graph is asymptotic to the line $y=-n \alpha^{-1} x-a_{n-1} a_{n}^{-1}$. Thus this line together with the lines $y=\beta_{i}$ (arising from $f^{\prime}\left(\beta_{i}\right)=0$ ) form the set of asymptotes and claim (4) holds. Since there are $s$ points $\beta_{i}$, claim (1) will follow from the arguments above and (3). Now consider the interval $\left(y_{1}\right.$, $y_{2}$ ) where $y_{1}$ and $y_{2}$ are two consecutive roots of $f^{\prime}$ which are not roots of $f$, or $y_{1}=-\infty$ if $f^{\prime}$ has no such root less than $y_{2}$, or $y_{2}=$ $\infty$ if $f^{\prime}$ has no such root greater than $y_{1}$. To prove claim (3), we need only consider the case where there exists a point $y_{0}$ in $\left(y_{1}, y_{2}\right)$ such that $f\left(y_{0}\right)=0$. Note that there can be at most one such point by the choice of $y_{1}$ and $y_{2}$ and Rolle's theorem. Assume first that $f^{\prime}\left(y_{0}\right) \neq 0$. Then $f^{\prime}$ has constant sign on $\left(y_{1}, y_{2}\right)$, so that $f$ is monotonic on the interval. Since $y_{0}$ is a simple root of $f$, equation (2.2) implies that $x$ ranges from $-\infty$ to $\infty$ (or vice versa) as $y$ ranges from $y_{1}$ to $y_{2}$. Thus this branch intersects each line $x=c$ where $c$ is an arbitrary constant. Now assume that $y_{0}$ is a root of $f$ of multiplicity $k$. The $k-1$ components $y=y_{0}$ satisfy (3). The remaining branch through $\left(0, y_{0}\right)$ behaves as in the case where $f^{\prime}\left(y_{0}\right)$ is nonzero since $f^{\prime}(y)$ can be factored as $\left(y-y_{0}\right)^{k-1}$ times a function 
which has constant sign on $\left(y_{1}, y_{2}\right)$ and $f(y)$ factors as $\left(y-y_{0}\right)^{k-1}$ times a polynomial which has a simple root at $y_{0}$. Since $f$ is monotonic on each subinterval $\left(y_{1}, y_{0}\right)$ and $\left(y_{0}, y_{2}\right)$, equation (2.2) implies that for this branch, $x$ ranges from $-\infty$ to $\infty$ (or vice versa) as $y$ ranges from $y_{1}$ to $y_{2}$. This concludes the proof of the lemma.

We now proceed to our main result. The main point of this theorem is to guarantee that no branch of $F(x, y)=0$ can pass through two distinct roots of $f(y)$ on the $y$-axis.

TheOREM 2.3. Given $h(x)=\sum_{i=0}^{n} b_{i} x^{i}, n \geqq 1, b_{0} \neq 0, b_{n}=1$, with only real roots and $f(y)$ an arbitrary polynomial, form $F(x, y)=$ $\sum_{i=0}^{n} b_{i} x^{i} f^{(i)}(y)$. Each branch of the real curve $F(x, y)=0$ which intersects the $y$-axis will intersect the $y$-axis in exactly one point and will intersect each vertical line $x=c$, where $c$ is an arbitrary constant. If $b_{0}=0$, the conclusion still holds for all branchs which do not coincide with the $y$-axis. Furthermore, if two branches which cross the $y$-axis intersect at a singular point $\left(x_{0}, y_{0}\right)$ not on the $y$-axis, then these branches are in fact components of the form $y-y_{0}=0$, and thus coincide as horizontal lines.

Proof. The theorem follows from the following somewhat stronger statement which we shall prove:

(2.4)(a). In the graph of $F(x, y)=0$, there does not exist a pair of disjoint paths from the $y$-axis to any point $\left(x_{0}, y_{0}\right)$ off the $y$-axis; (We say two paths are disjoint if their interiors have no point in common.)

(2.4)(b). Two coincidental paths from the $y$-axis to a point $\left(x_{0}, y_{0}\right)$ with $x_{0} \neq 0$ are necessarily horizontal lines $y=y_{0}$;

(2.4)(c). Multiple straight line components can intersect no branches off the $y$-axis that are not coincidental straight lines.

If $b_{0}=0$, let $b_{r}, r>0$, be the first nonzero coefficient in $h$ and write $g(y)=f^{(r)}(y)$. Then $F(x, y)=x^{r} \sum_{i=0}^{n-r} b_{i+r} x^{i} g^{(i)}(y)$ has $r$ components which coincide with the $y$-axis and the new polynomials which replace $h$ and $f$ satisfy the hypotheses of the theorem. Thus we may assume $b_{0} \neq 0$.

Note that it suffices to prove statement (2.4) for points with $x_{0}>0$. For if we replace $h(x)$ by $h(-x)$, these points are reflected about the $y$-axis and the new polynomial $h$ will still have only real roots. 
We now prove the theorem by induction on $n$, the degree of $h$. If $n=1$, the conditions of (2.4) follow from Lemma 2.1. Now assume $n>1$ and that (2.4) holds for polynomials $h$ of degree less than $n$ and arbitrary polynomials $f$. Assume now that $h$ has degree $n$. Since $h$ has only real roots, we can factor $h(x)=\left(x+\alpha_{1}\right) \cdots$ $\left(x+\alpha_{n}\right)$. Let $\alpha=\alpha_{n}$ and $g(x)=\left(x+\alpha_{1}\right) \cdots\left(x+\alpha_{n-1}\right)$, so that $h(x)=$ $(x+\alpha) g(x)$. Write $g(x)=\sum_{i=0}^{n-1} d_{i} x^{i}$; the induction hypothesis says that the real algebraic curve

$$
F_{n-1}(x, y)=d_{0} f(y)+d_{1} x f^{\prime}(y)+\cdots+d_{n-1} x^{n-1} f^{(n-1)}(y)=0
$$

satisfies statement (2.4).

Next we define a variety in three variables by

$$
G(x, y, t)=\alpha F_{n-1}(t, y)+x \frac{\partial}{\partial y} F_{n-1}(t, y)=0 .
$$

For any fixed value of $t=t_{0}$, we can apply Lemma 2.1 to $G\left(x, y, t_{0}\right)$ where the polynomial in question is $F_{n-1}\left(t_{0}, y\right)$. In particular, the intersection of each plane $t=t_{0}$ with the variety $G=0$ satisfies condition (2.4) with the $y$-axis replaced by the line $x=0=t-t_{0}$. We shall refer to this algebraic curve as $G_{t}(x, y)=0$. For any fixed value of $x=x_{0}$, we obtain

$$
\begin{aligned}
G\left(x_{0}, y, t\right) & =\alpha F_{n-1}(t, y)+x_{0} \frac{\partial}{\partial y} F_{n-1}(t, y) \\
& =\sum_{i=0}^{n-1} d_{i} t^{i} \frac{\partial^{i}}{\partial y^{i}}\left(\alpha f(y)+x_{0} f^{\prime}(y)\right) .
\end{aligned}
$$

By applying the induction hypothesis to $g(t)$ and the polynomial $\alpha f(y)+x_{0} f^{\prime}(y)$, we see that the intersection of each plane $x=x_{0}$ with the variety $G=0$ also satisfies condition (2.4) in the variables $t$ and $y$ with the $y$-axis replaced by the line $x-x_{0}=0=t$. We shall refer to this curve as $F_{n-1, x_{0}}(t, y)=0$. Finally, we consider the intersection of $G=0$ with the plane $x=t$, and note that a straightforward computation shows that $G(x, y, x)=F_{n}(x, y)$ for all values of $x$ and $y$, where $F_{n}(x, y)=F(x, y)=0$ is the curve involving the polynomial $h(x)$ that the statement of the theorem is concerned with. Thus it will suffice to show that the curve obtained from the intersection of $G=0$ with the plane $x=t$ satisfies condition (2.4). We identify the curve in the plane $x=t$ with the variety $F_{n}=0$ and the curve $F_{n-1,0}$ in the $y t$-plane with the curve $F_{n-1}=0$. For the remainder of this proof, we shall write " $y$-axis" in quotes to mean the appropriate line in any of the above planes with $x$ or $t$ constant, for which we know that the curve induced by $G=0$ satisfies condition (2.4). 
Now we assume that condition $(2.4)(a)$ is violated by the curve $F_{n}=0$, but $(2.4)(\mathrm{b})$ and $(2.4)(\mathrm{c})$ both hold. Choose paths $P_{1}$ and $P_{2}$ in the $x=t$ plane which give the contradiction to the theorem in such a way that the area enclosed by the paths and the $y$-axis is minimal; in particular, the points $y_{1} \geqq y_{2}$ where the paths intersect the $y$-axis are consecutive roots of $f$. We also allow the possibility that the paths begin at a multiple root $y_{1}=y_{2}$ of $f$ with the path $P_{1}$ through $y_{1}$ having greater $y$-values for $x$ near zero than the path $P_{2}$ through $y_{2}$. We may also assume that $\left(x_{0}, y_{0}\right)$ is a point for which $x_{0}$ is the maximum value taken on by $x$ in both paths.

We shall consider the algebraic curve $G_{t}=0$ for each fixed $t$. The points of the paths $P_{i}$ are obtained from the intersection of the branches of $G_{t}$ with the plane $x=t$. Unfortunately, these branches do not always vary continuously with $t$. The following lemma describes how discontinuities can occur.

Lemma 2.7. Set $f_{t}(y)=F_{n-1}(t, y)$, so that for fixed $t$ equation (2.5) becomes

$$
G_{t}(x, y)=\alpha f_{t}(y)+x f_{t}^{\prime}(y)
$$

In $G_{t}(x, y)=0$, the partition of the points of the curve into branches for fixed $t$ varies continuously with $t$ except possibly at a point $t=s$ such that $f_{s}$ or $f_{s}^{\prime}$ has a multiple real root.

(a) If $f_{s}$ has a double real root at $y_{0}$, then for values of $t$ near $s$, the curve $G_{t}(x, y)$ may have two branches crossing the $y$-axis near $y_{0}$ or may have two branches coming close to $\left(0, y_{0}\right)$ and contained entirely on opposite sides of the $y$-axis. If $f_{\mathrm{s}}$ has a root $y_{0}$ of multiplicity $m>2$, then for values of $t$ near $s$, the curve $G_{t}$ has $r \leqq m$ branches crossing the $y$-axis near $y_{0}$; the number $m-r$ is even; and there are at most $(m-r) / 2$ branches contained entirely in the half plane $x>0$.

(b) If $f_{s}^{\prime}$ has a real root of multiplicity $m>1$ at $y_{0}$ with $f_{s}\left(y_{0}\right) \neq 0$, then the curve $G_{s}$ has a (multiple) horizontal asymptote $y=y_{0}$. For nearby values of $t$, the asymptotes can separate, creating new branches (not crossing the y-axis) or disappear in pairs, causing adjacent branches which were separated by an even number of asymptotes to join into a single branch. (We shall refer to this as "joining at infinity.") Note that $f_{t}^{\prime}$ must have an odd number of roots between any two consecutive roots of $f_{t}$, so two branches crossing the $y$-axis cannot join at infinity.

Proof. The lemma follows almost immediately from Lemma 2.1 and Rolle's theorem. By Lemma 2.1, two branches can only meet 
at the $y$-axis, corresponding to a multiple root of $f_{t}$, or at infinity, corresponding to a multiple asymptote of even multiplicity (i.e., a multiple real root of $f_{t}^{\prime}$ ). Thus discontinuities can only occur in cases (a) and (b). The analysis of the individual cases is clear from Lemma 2.1 and a consideration of how multiple roots of a polynomial and its derivative may vary as the polynomial varies continuously.

We now continue the proof of the theorem. Assume first that $y_{1}=y_{2}$ and, as $t$ increases, the branches of $G_{t}$ which generate the paths in $F_{n}$ trace out straight lines $y=y_{1}=y_{2}$ in $F_{n-1}$. For the two paths $P_{1}$ and $P_{2}$ to meet at $\left(x_{0}, y_{0}, x_{0}\right)$, the corresponding branches of $G_{t}$ must vary to become a pair of straight lines $y=y_{0}=y_{1}$ in $G_{x_{0}}(x, y)=0$; but then Lemma 2.1 implies that there is at least one more branch of $G_{x_{0}}$ passing through $\left(0, y_{0}, x_{0}\right)$. Thus the straight lines in $F_{n-1}$ meet another branch at $\left(0, y_{0}, x_{0}\right)$, in contradiction to $(2.4)(c)$. Therefore, using (2.4)(b) and (c), we can conclude that as $t$ increases from zero, the branches of $G_{0}$ through $y_{1}=y_{2}$ must separate, continuing to cross the $y$-axis, for small values of $t$, at the points where the branches of $F_{n-1}$ through $y_{1}=y_{2}$ meet the constant $t$ planes.

As we analyze the behavior of the branches of $G_{t}$, we shall be looking for a contradiction in one of the curves $F_{n-1, c}$ where $c$ is a constant with $0 \leqq c \leqq x_{0}$. The branches of $G_{0}$ passing through $y_{1}$ and $y_{2}$ give points $\left(c, \bar{y}_{i}(c), 0\right)$ in the plane $x=c$ for $i=1,2$, respectively. If there is more than one choice for $\bar{y}_{i}(c)$, let $\bar{y}_{i}(c)$ denote the set of $y$-values in the plane $x=c$ corresponding to $y_{i}$.

From equation (2.5) and the induction hypothesis on $F_{n-1}$, we see that the branches through $y_{1}$ and $y_{2}$ in $G_{0}$ cannot continuously vary to join (or rejoin if $y_{1}=y_{2}$ ) each other at the " $y$-axis" as $t$ increases, for this would create a path in $F_{n-1}$ contradicting the induction hypothesis. By Lemma 2.7(b), they also cannot join at infinity since there are an odd number of roots of $f^{\prime}$ between $y_{1}$ and $y_{2}$ by Rolle's theorem. On the other hand, they must somehow vary so that the two paths in $F_{n}$ meet at $\left(x_{0}, y_{0}, x_{0}\right)$, which must be a point of $G_{x_{0}}$ at which $(\partial / \partial y) G_{x_{0}}=0$. By Lemma 2.1, this point will either lie on a single branch of $G_{x_{0}}$ or will lie on a set of multiple straight lines $y=y_{0}$ (in the plane $t=x_{0}$ ). Thus the branches of $G_{0}$ through $y_{1}$ and $y_{2}$ must first join with other branches (which did not cross the $y$-axis at $t=0$ or else this would again lead to a contradiction in $F_{n-1}$ ).

The analysis of what happens using Lemma 2.7, especially with regard to behavior at infinity, requires that we know the sign of $\alpha$, which determines the slopes of the branches of $G_{t}$ at the " $y$ - 
axis." Henceforth, we shall assume $\alpha>0$. The analysis for $\alpha<0$ is similar and will be omitted. Since $\alpha>0$, branches can only be joined at infinity if the lower one does not cross the $y$-axis. If such a branch lies between the branch through $y_{1}$ and the branch through $y_{2}$, then joining it to the branch through $y_{1}$ has essentially no effect, since the branch through $y_{1}$ could have been continuously varied to obtain the same result. So let $\left(0, \widetilde{y}, t_{0}\right)$ be the point on the " $y$-axis" at which two branches of $G_{t}$ come to the " $y$-axis," or appear as a pair of straight lines through a root of $f_{t}$ as in Lemma 2.7(a), prior to at least one of them joining with one of our original branches. Let $y_{i}(t)$ denote the $y$-coordinate at $x=0$ of the branch of $G_{t}$ continuously obtained from the original branch through $y_{i}$; this is well defined for $t>0$ until the first time $y_{i}(t)$ is a multiple root of $f_{t}$. Then we have three possibilities:

$$
\begin{aligned}
y_{1}\left(t_{0}\right) & >\widetilde{y}>y_{2}\left(t_{0}\right) ; \\
\widetilde{y} & \geqq y_{1}\left(t_{0}\right) ; \\
\widetilde{y} & \leqq y_{2}\left(t_{0}\right) .
\end{aligned}
$$

We consider the effect of each of these possibilities and show that no combination of them can allow the paths in $F_{n}$ to occur without giving rise to a contradiction in one of the curves $F_{n-1, c}$, where $0 \leqq c \leqq x_{0}$, which, as we have seen, must satisfy the induction hypothesis.

Assume first that situation (A) occurs alone. Then the only way to have the paths $P_{1}$ and $P_{2}$ meet each other is to have one of the new real roots of $f_{t}$ vary to meet $y_{1}(t)$ and the other to meet $y_{2}(t)$, and then subsequently joining these branches. (Note that connections at infinity cannot be used since they require that at least one branch being joined not cross the $y$-axis. Also the polynomial $f_{t}^{\prime}$ has an odd number of roots between $y_{1}(t)$ and $y_{2}(t)$.) But we obtain a contradiction as soon as both of the new branches have joined to the original ones since this generates a path in $F_{n-1}$ from $\left(0, y_{1}\right)$ to $\left(0, y_{2}\right)$ passing through the point $\left(t_{0}, \widetilde{y}\right)$. Indeed, more than one application of (A) can do no better; to avoid leaving a line between the branches and thus separating $P_{1}$ and $P_{2}$, the new branches all must eventually join (prior to or at $t=x_{0}$ ) to induce a contradiction in the curve $F_{n-1}$.

No branch can connect via infinity with the branch through $y_{1}(t)$ since $\alpha>0$ (see equation (2.2)). Now we consider the effect of situation (B). The new branch must meet $y_{1}(t)$ and pull off the $y$-axis as $t$ increases. The new branch $\beta$ thus formed cannot join at infinity with the branch coming from $y_{2}$ (again because $\alpha>0$ ), hence to obtain the connection of $P_{1}$ and $P_{2}$, the branches must 
rejoin the " $y$-axis" and part again. The lower one must then join either with the branch through $y_{2}(t)$ or with the upper branch from situation (A), while the lower branch joins with the branch through $y_{2}(t)$. (As noted above, applications of (A) have no substantial effect on the argument.) This use of (B) prevents a contradiction from occuring in $F_{n-1}$ by breaking the path which would otherwise be generated from $y_{1}$ to $y_{2}$. But, if we let $x_{m}$ be the maximum value of $x$ to which the branch $\beta$ pulls away from the $y$-axis, say at $t=t_{m}$, and note that $x_{m} \leqq x_{0}$ since a point is being generated on $P_{1}$ by this branch, then the contradiction is to be found in the plane $x=x_{m}$. Indeed, in the curve $F_{n-1, x_{m}}$, a path will be generated between the smallest value of $\bar{y}_{1}\left(x_{m}\right)$ and the largest value of $\bar{y}_{2}\left(x_{m}\right)$ on the " $y$-axis" since in this plane it is not broken, but has a singularity at $t=t_{m}$. (The only way this could be avoided is by having some other path joining two points of $\bar{y}_{1}\left(x_{m}\right)$ or two points of $\bar{y}_{2}\left(x_{m}\right)$, which again would contradict the induction hypothesis.) If more than one application of situation (B) occurs, the largest value of $x_{m}$ determines the plane in which a contradiction occurs. Thus situation (B) has virtually no effect other than to shift the plane in which a path is generated which violates the induction hypothesis.

Situation (C) leads to a contradiction also as for (B) (or any combination of (A), (B), and (C)) if the branches which leave the $y$-axis later return to it as they had to for (B). The other possibility is that they do not return to the $y$-axis, but rather connect with a branch above (i.e., larger $y$-values: either a branch through $y_{1}(t)$ or one coming from applying (A) or (B)) by joining at infinity. In this case the contradiction always occurs in $F_{n-1, x_{0}}$ since, if no other contradiction occurs with the points of $\bar{y}_{i}\left(x_{0}\right), i=1,2$, then the paths through the smallest value of $\bar{y}_{1}\left(x_{0}\right)$ and the largest value of $\bar{y}_{2}\left(x_{0}\right)$ will meet at $\left(x_{0}, y_{0}, x_{0}\right)$. The meeting occurs either as the branch in question pulls back from infinity to $x_{0}$ (i.e., the point at which $(\partial / \partial y) G_{t}=0$ on the branch is the point $\left(x_{0}, y_{0}\right)$ when $\left.t=x_{0}\right)$; or, if this happens for $t<x_{0}$, then necessarily (A) has occured and another point of $G_{t}$ with $(\partial / \partial y) G_{t}=0$ will be the last to pull back to $x=x_{0}$ at $t=x_{0}$. Note that if $\alpha<0$, the arguments for cases (B) and (C) must be reversed. This completes the proof of (2.4)(a).

Next we prove $(2.4)(\mathrm{b})$ and $(2.4)(\mathrm{c})$ in a similar manner. For (2.4)(b), assume that $F_{n}$ has two coincidental paths from a point $y_{0}$ on the $y$-axis. Then for each $t>0$, there exists a pair of straight lines in $G_{t}$ which generate the path. These pairs of straight lines generate coincidental paths in $F_{n-1}$, so by the induction hypothesis the paths in $F_{n-1}$ are horizontal straight lines. But then we must have straight lines in $F_{n}$ also. Finally, for $(2.4)(\mathrm{c})$, we assume that 
$F_{n}$ has $m$ multiple straight lines $y=y_{0}$ which intersect some other branch of $F_{n}$ at $t=t_{0}>0$. Then, as above, we obtain $m$ straight lines $y=y_{0}$ in each $G_{t}, 0 \leqq t<t_{0}$, and at least $m+1$ lines in $G_{t_{0}}$. By Lemma 2.1(2), these generate $m+1$ straight lines $y=y_{0}$ in $F_{n-1}$ which intersect another branch of $F_{n-1}$ at $\left(t_{0}, y_{0}\right)$, contradicting the induction hypothesis. This completes the proof of the theorem.

REMARK 2.9. All of the complications in the preceding proof are caused by the fact that it is possible to have branches in $F_{n-1}$ which cross the $y$-axis and have more than one point with the same $x$-coordinate. This sometimes happens when the degree of $h$ is less than the degree of $f$. We conjecture that this does not happen if the degree of $h$ is greater than or equal to the degree of $f$; it certainly cannot happen if $f$ has only real roots, for then a vertical line would intersect the curve in more points than the degree. An interesting example in this regard is obtained by taking $h(x)$ to be a power of $x+1$ and $f(y)=11-16 y+8 y^{2}-4 y^{3}+y^{4}$ (see Figure 2.1). Then using the notation of the proof above, the curve $F_{2}(x, y)$ has a branch which intersects some vertical lines in three points, but no other $F_{n}$ has such a branch. The curve $F_{4}$ is also interesting; it comes very close to having a singularity near $(.6,-1.5)$ causing one of the branches to intersect some horizontal lines in three points.
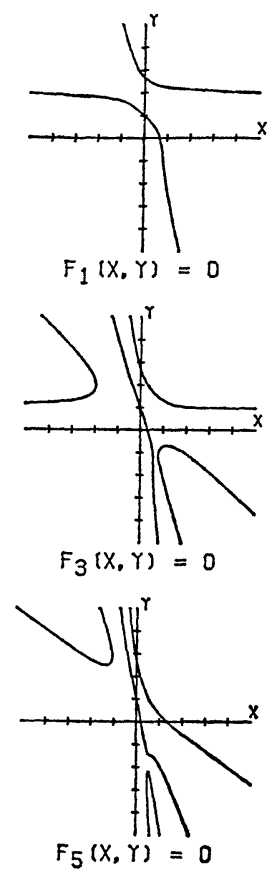

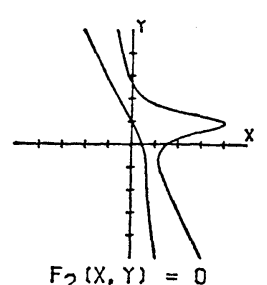

$F_{2}(X, Y)=0$

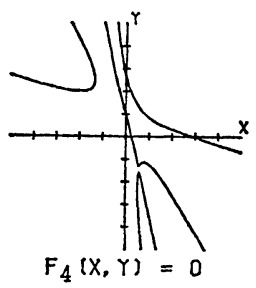

Figure 2.1. 
Also of interest is the fact that, in going from $F_{2}$ to $F_{3}$, the number of branches increases from two to four.

3. Applications. We begin with a direct extension of Theorem 1.1 of Pólya to arbitrary polynomials $f$.

THEOREM 3.1. Let $h(x)=\sum_{i=0}^{n} b_{i} x^{i}$ be a polynomial of degree $n$ with only real negative roots and $f(y)$ an arbitrary polynomial with $r$ real roots and degree at most $n$. The real algebraic curve $F(x, y) \equiv \sum_{i=0}^{n} b_{i} x^{i} f^{(i)}(y)=0$ has at least $r$ intersection points with each line $s x-t y+u=0$ where $s \geqq 0, t \geqq 0, s+t>0$ and $u$ is real.

Proof. Write $f(y)=\sum_{i=0}^{m} a_{i} y^{i}, a_{m} \neq 0, m \leqq n$, and set $y=t x$ in the equation for the curve $F(x, y)=0$; compute

$$
\begin{gathered}
a_{m}^{-1} \lim _{|x| \rightarrow \infty} x^{-m} F(x, t x)=b_{0} t^{m}+m b_{1} t^{m-1}+m(m-1) b_{2} t^{m-2} \\
+\cdots+m ! b_{m} .
\end{gathered}
$$

We claim that this polynomial has only real negative roots. By hypothesis $h(t)=\sum b_{i} t^{i}$ has only real negative roots, and therefore

$$
g(t)=b_{0}+b_{1} t+\left(1-\frac{1}{m}\right) b_{2} t^{2}+\cdots+\left(1-\frac{1}{m}\right) \cdots\left(1-\frac{m-1}{m}\right) b_{m} t^{m}
$$

has only real negative roots by [4, p, 343]. But then $t^{m} g(m / t)$, which equals the polynomial in (3.2), has only real negative roots. This implies that each branch of $F(x, y)=0$ is asymptotic to lines with negative slopes, the possible slopes being given by the values of $t$ which make the expression in (3.2) equal to zero. For each of the $r$ real roots of $f$, Theorem 2.3 implies that there is a branch of $F(x, y)$ which passes through the root on the $y$-axis and for which $x$ goes from $-\infty$ to $+\infty$ as $y$ goes from $+\infty$ to $-\infty$ since the asymptotes have negative slopes. Thus by continuity each of the branches must intersect every line with zero, positive or infinite slope.

For many applications, we prefer to relax the conditions on $h(x)$ slightly and lose some lines in the conclusion. Note that we remove the restriction on the degree of $f$.

THEOREM 3.3. Let $h(x)=\sum_{i=0}^{n} b_{i} x^{i}$ be a polynomial of degree $n$ with only real nonpositive roots and $f(y)$ an arbitrary polynomial with $r$ real roots. The real algebraic curve $F(x, y) \equiv \sum_{i=0}^{n} b_{i} x^{i} f^{(i)}(y)=$ 0 has at least $r$ intersection points with every line of positive slope. 
Proof. Assume first that zero is not a root of $h$. Then the only difference in hypotheses from the previous theorem is that the degree of $f$ may be greater than the degree of $h$. The proof is thus the same as the previous proof, except that the polynomial in equation (3.2) may have zero as a root. That is, the branches of $F(x, y)$ may have horizontal asymptotes. Hence lines of zero slope may miss the branches, but lines of positive slope will still intersect any branch which crosses the $y$-axis.

If zero is a root of $h$, let $b_{s}, s>0$, be the first nonzero coefficient of $h$ and write $g(y)=f^{(s)}(y)$. Note that $g$ has at least $r-s$ real roots by Rolle's theorem. Then $F(x, y)=x^{s} \sum_{i=0}^{n-s} b_{i+8} x^{i} g^{(i)}(y)$ has $s$ components coinciding with the $y$-axis and the remainder of the curve has at least $r-s$ intersections with each line of positive slope. Since every line of positive slope must cross the $y$-axis, the theorem holds.

We can now obtain the corollaries mentioned in the introduction. The first is a corollary of Theorem 2.3.

Corollary 3.4 (Hermite-Poulain). Let $f(x)$ be a polynomial with real coefficients and $h(x)=b_{0}+b_{1} x+\cdots+b_{n} x^{n} a$ polynomial with only real roots. Then the polynomial $g(x)=\sum_{i=0}^{n} b_{i} f^{(i)}(x)$ has at least as many real roots as $f$. If $f$ has only real roots, then every multiple root of $g$ is also a multiple root of $f$.

Proof. The real curve $F(x, y) \equiv \sum_{i=0}^{n} b_{i} x^{i} f^{(i)}(y)=0$ has a branch crossing the $y$-axis for each real root of $f$. By Theorem 2.3, these branches intersect the line $x=1$. The polynomial $\sum_{i=0}^{n} b_{i} f^{(i)}(y)$ has a root for each intersection, and therefore has at least as many real roots as $f$. If $f$ has only real roots, then every branch of $F(x, y)$ intersects the $y$-axis. If $g$ has a multiple root $c$, then $F(x$, $y$ ) has a singular point at $(1, c)$ where two branches intersect. By the last assertion of Theorem 2.3, these branches are horizontal lines, so that $c$ is also a multiple root of $f$.

CoROLlary 3.5. Let $h(x)=\sum_{i=0}^{n} b_{i} x^{i}$ be a polynomial of degree $n$ with only real roots, all of the same sign or zero, and let $f(x)=$ $\sum_{i=0}^{m} a_{i} x^{i}, m \leqq n$. Then the polynomial $\sum_{i=0}^{m} i ! a_{i} b_{i} x^{i}$ has at least as many real roots as $f$.

Proof. If the roots of $h$ are all negative, we set $y=0$ in Theorem 3.1 and note that $f^{(i)}(0)=i ! a_{i}$. If the roots of $h$ are all positive, replace $h(x)$ by $h(-x)$, apply the theorem, and then switch the variable again. If $h(x)=x^{s} \sum_{i=0}^{n-s} b_{i+s} x^{i}, b_{s} \neq 0$, then let $g(y)=$ 
$f^{(s)}(y)$ and apply Theorem 3.1 to $\sum b_{i+s} x^{i} g^{(i)}(y)$, setting $y=0$. By Rolle's theorem, the number of real roots of $f$ is at most equal to $s$ plus the number of real roots of $g$. Thus the conclusion again holds.

A slight change in the hypotheses of this corollary leads us to Corollary 3.6 in which we obtain the result separately for the positive and negative roots. In [2], Corollary 3.6 is used to solve an open problem of Karlin.

CoRollary 3.6. Let $h(x)=\sum_{i=0}^{n} b_{\imath} x^{i}$ be a polynomial of degree $n$ with only real negative roots and let $f(x)=\sum_{i=0}^{m} a_{i} x^{i}, m \leqq n$. Then the polynomial $\sum_{i=0}^{m} i ! a_{\imath} b_{i} x^{i}$ has at least as many positive roots as $f$ has positive roots and at least as many negative roots as $f$ has negative roots. The multiplicity of zero as a root is the same.

Proof. This follows from the proof of Theorem 3.1; since the asymptotes all have negative slopes, any branch which crosses the $y$-axis at a positive root of $f$ will cross the $x$-axis at a positive root of $\sum i ! a_{i} b_{i} x^{i}$, and similarly for negative roots.

CoRollary 3.7. Let $h(x)=\sum_{i=0}^{n} b_{i} x^{i}$ be a polynomial with only real nonpositive roots and let $f$ be an arbitrary polynomial with real coefficients. Then the polynomial $\sum_{i=0}^{n} b_{i} x^{i} f^{(i)}(x)$ has at least as many real roots as $f$.

\section{Proof. Set $y=x$ in Theorem 3.3.}

This corollary is the starting point for our work in [1], where it is used to obtain many results concerning polynomials and entire functions.

\section{REFERENCES}

1. T. Craven and G. Csordas, An inequality for the distribution of zeros of polynomials and entire functions, Pacific J. Math., 95 (1981), 263-280.

2. - Zero-diminishing linear transformations, Proc. Amer. Math. Soc., 80 (1980), 544-546.

3. J. Coolidge, A Treatise on Algebraic Plane Curves, Dover, New York, 1959.

4. B. Ja. Levin, Distribution of zeros of entire functions, Amer. Math. Soc. Transl., Providence, R.I., 1964.

5. N. Obreschkoff, Verteilung und Berechnung der Nullstellen Reeler Polynome, Veb Deutscher Verlag der Wissenschaften, Berlin, 1963.

6. G. Pólya, Über algebraische Gleichungen mit nur reelen Wurzeln, Vierteljschr. Naturforsch. Ges. Zürich, 61 (1916), 546-548.

7. G. Pólya and J. Schur. Uber zwei Arten von Faktorenfolgen in der Theorie der algebraischen Gleichungen, J. Reine Angew. Math., 144 (1914), 89-113. 
8. R. Walker, Algebraic Curves, Dover, New York, 1950.

Received May 19, 1981. The first auther was partially supported by NSF grant MCS79-00318.

UNIVERSITY OF HAWAII AT MANOA

HONOLULU, HI 96822 


\section{PACIFIC JOURNAL OF MATHEMATICS}

\section{EDITORS}

DONALD BABBITT (Managing Editor)

University of California

Los Angeles, California 90024

HUGO ROSSI

University of Utah

Salt Lake City, UT 84112

C. C. MOORE and ARTHur AGUS

University of California

Berkeley, CA 94720
J. DUGUNDJI

Department of Mathematics

University of Southern California

Los Angeles, California 90007

R. FINN and J. MILGRAM

Stanford University

Stanford, California 94305

\section{ASSOCIATE EDITORS}
R. Arnes
E. F. BECKENBACH
B. H. NeumanN
F. WOLF
K. YösHIDA

\section{SUPPORTING INSTITUTIONS}

UNIVERSITY OF ARIZONA

UNIVERSITY OF BRITISH COLUMBIA

CALIFORNIA INSTITUTE OF TECHNOLOGY

UNIVERSITY OF CALIFORNIA

MONTANA STATE UNIVERSITY

UNIVERSITY OF NEVADA, RENO

NEW MEXICO STATE UNIVERSITY

OREGON STATE UNIVERSITY
UNIVERSITY OF OREGON

UNIVERSITY OF SOUTHERN CALIFORNIA

STANFORD UNIVERSITY

UNIVERSITY OF HAWAII

UNIVERSITY OF TOKYO

UNIVERSITY OF UTAH

WASHINGTON STATE UNIVERSITY

UNIVERSITY OF WASHINGTON 


\section{Pacific Journal of Mathematics}

Vol. 102, No. $1 \quad$ January, 1982

S. Agou, Degré minimum des polynômes $f\left(\sum_{i=0}^{m} a_{i} X^{p^{r i}}\right)$ sur les corps finis

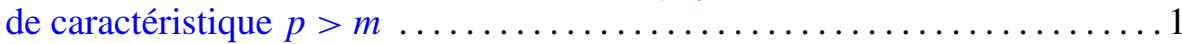

Chi Cheng Chen, On the image of the generalized Gauss map of a complete

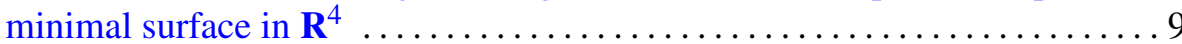

Thomas Curtis Craven and George Leslie Csordas, On the number of real

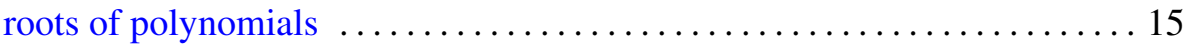

Allan L. Edelson and Kurt Kreith, Nonlinear relationships between oscillation and asymptotic behavior ....................... 29

B. Felzenszwalb and Antonio Giambruno, A commutativity theorem for

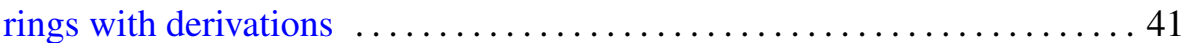

Richard Elam Heisey, Manifolds modelled on the direct limit of lines . . . . . 47

Steve J. Kaplan, Twisting to algebraically slice knots $\ldots \ldots \ldots \ldots \ldots \ldots 5$

Jeffrey C. Lagarias, Best simultaneous Diophantine approximations. II.

Behavior of consecutive best approximations $\ldots \ldots \ldots \ldots \ldots \ldots \ldots 61$

Masahiko Miyamoto, An affirmative answer to Glauberman's conjecture . . . 889

Thomas Bourque Muenzenberger, Raymond Earl Smithson and L. E.

Ward, Characterizations of arboroids and dendritic spaces ........... 107

William Leslie Pardon, The exact sequence of a localization for Witt

groups. II. Numerical invariants of odd-dimensional surgery

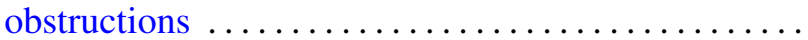

Bruce Eli Sagan, Bijective proofs of certain vector partition identities

Kichi-Suke Saito, Automorphisms and nonselfadjoint crossed products ...

John Joseph Sarraille, Module finiteness of low-dimensional PI rings ...

Gary Roy Spoar, Differentiable curves of cyclic order four . .

William Charles Waterhouse, Automorphisms of quotients of $\Pi \mathrm{GL}\left(n_{i}\right)$

Leslie Wilson, Mapgerms infinitely determined with respect to right-left equivalence

Rahman Mahmoud Younis, Interpolation in strongly logmodular

algebras 